\title{
Catalisadores Sulfônicos Imobilizados em Polímeros: Síntese, Caracterização e Avaliação
}

\author{
Fernanda M.B. Coutinho \\ Instituto de Química, UERJ
}

\section{Simone M. Rezende \\ Instituto de Macromoléculas Professora Eloisa Mano, UFRJ}

Resumo: Suportes poliméricos à base de estireno (Sty) e divinilbenzeno (DVB) foram preparados por polimerização em suspensão aquosa em presença de diluente (mistura de tolueno e n-heptano em composição variada) em um grau de diluição de $100 \%$ e com quatro teores diferentes de DVB (10, 20, 40 e $60 \%$ ). Os suportes foram caracterizados nos estados seco e inchado. No estado seco foram analisados quanto à densidade aparente, área específica, volume de poros e morfologia, e no estado inchado foi observado o comportamento em dois diferentes tipos de solventes, um bom e um mau solvente para o copolímero de estirenodivinilbenzeno. As propriedades dos suportes poliméricos, nos estados seco e inchado, foram relacionadas com os parâmetros de síntese. Os suportes poliméricos foram sulfonados em presença de solvente capaz de inchar a rede polimérica (1,2-dicloroetano). Os catalisadores sulfônicos obtidos foram caracterizados no estado seco e inchado e a atividade catalítica foi avaliada em reações de esterificação de ácido acético com nbutanol. Também foi avaliado o efeito da reciclagem dos catalisadores na sua atividade catalítica.

Palavras-chave: Suportes poliméricos de estireno-divinilbenzeno, morfologia, caracterização, catalisadores sulfônicos imobilizados, reação de esterificação.

\section{Polymer Supported Sulfonic Catalysts: Synthesis, Characterization and Evaluation}

Abstract: Polymer supports based on styrene and divinylbenzene were prepared by aqueous suspension polymerization in presence of diluents (mixtures of toluene and heptane in varied compositions) at $100 \% \mathrm{v} / \mathrm{v}$ of dilution degree (relative to the monomers volume) at four DVB proportions (10, 20, 40 and 60\%). The supports were characterized in the dry and swollen states. In the dry state, the apparent density, surface area, pore volume and morphology were determined. In the swollen state, the behavior in two different solvents, one good and one bad for the styrene-divinylbenzene copolymer, was observed. The polymer support properties in the dry and swollen states were related with the respective synthesis parameters. The polymer supports were sulfonated in the presence of a solvent able to swell the polymer network (1,2-dichloroethane). The obtained supported catalysts were also analyzed in the dry and swollen states. The catalysts activities were evaluated in the acetic acid esterification with n-butanol, and the recycling effect of two different catalysts on its activities was also evaluated.

Keywords: Styrene-divinylbenzene polymer supports, synthesis, characterization, polymer supported sulfonic catalysts, catalyst recycling.

\section{Introdução}

Resinas de troca iônica podem ser definidas como matrizes poliméricas insolúveis contendo grupos ionizáveis fixos em sua estrutura, através dos quais pode ocorrer a troca iônica. As cargas fixas no esqueleto polimérico podem ser positivas ou negativas, conferindo, assim, à resina a capacidade de trocar

Autor para correspondência: Fernanda M.B. Coutinho, Instituto de Macromoléculas Professora Eloisa Mano, UFRJ, C.P. 68525, CEP: 21945-970, Rio de Janeiro, RJ.E-mail:fern@ima.ufrj.br 
ânions ou cátions, respectivamente ${ }^{[1]}$. Essas matrizes poliméricas funcionalizadas têm se tornado muito importantes, como suportes, em muitos campos da pesquisa científica, bem como em aplicações industriais $^{[2]}$.

Entre as matrizes poliméricas mais utilizadas, estão as produzidas pela copolimerização em suspensão aquosa de estireno (Sty) e divinilbenzeno (DVB). Entre as modificações químicas mais empregadas nesses materiais, está a funcionalização com grupos ácido sulfônico, dando origem a catalisadores ácidos. Essas resinas de troca iônica, particularmente as macroporosas, desempenham um papel muito importante como catalisadores heterogêneos, em relação aos sistemas catalíticos homogêneos ${ }^{[3,4]}$, tanto do ponto de vista da catálise, como do ponto de vista da engenharia das reações para fins comerciais. Além disso, os catalisadores imobilizados em polímeros podem ser reciclados e, freqüentemente, levam a reações com alta seletividade ${ }^{[5,6]}$. A matriz polimérica (resina) permite que as reações sejam conduzidas tanto em meio aquoso como em meio orgânico polar ou $\operatorname{apolar}^{[6]}$.

Os trabalhos desenvolvidos até agora, com o objetivo de investigar as propriedades catalíticas de grupos ácido sulfônico imobilizados em copolímeros reticulados de Sty-DVB, mostraram claramente que a atividade catalítica e a seletividade desses sistemas dependem de parâmetros diferentes que estão interligados de maneira complexa. Além da estrutura e densidade de ligações cruzadas da rede polimérica, as propriedades catalíticas são influenciadas pela distribuição e concentração local dos grupos ácido sulfônico $\left(-\mathrm{SO}_{3} \mathrm{H}\right)$ na rede macromolecular ${ }^{[3]}$.

Entre as características morfológicas que influenciam a atividade do catalisador sulfônico imobilizado em polímero estão a área específica, o volume de poros, o diâmetro médio de poro e o inchamento percentual nas condições reacionais. Uma variedade de catalisadores pode ser sintetizada pela variação dos parâmetros reacionais na produção de resinas utilizadas como catalisadores. As características morfológicas desses suportes poliméricos são controladas pelas variáveis do processo, principalmente, pela relação molar entre o estireno e o divinilbenzeno, pela presença ou não de agente porogênico e pelo grau de diluição da mistura monomérica ${ }^{[4,7-9]}$.

O presente trabalho trata do preparo, da caracterização e da avaliação em reação de esterificação, de catalisadores sulfônicos imobilizados em pérolas de poli(estireno-co-divinilbenzeno), tendo, como centros ativos, grupos sulfônicos, introduzidos por meio de reação de sulfonação da matriz polimérica.

\section{Matériais e Métodos}

\section{Síntese do suporte polimérico (Sty/DVB)}

Os suportes poliméricos de Sty/DVB foram sintetizados por meio de copolimerização em suspensão aquosa, em balão de três bocas de fundo redondo, de $1000 \mathrm{ml}$ de capacidade, equipado com agitador mecânico e condensador de refluxo. Para estabilizar a suspensão utilizou-se hidroxetilcelulose (HEC a $0,2 \%$ p/v em relação à água) e cloreto de sódio $(\mathrm{NaCl}$ $0,5 \%$ p/v em relação à água) foi empregado para diminuir a solubilidade dos monômeros na água. $\mathrm{O}$ iniciador utilizado foi o peróxido de benzoíla (BPO) em concentração igual a $1 \%$ em relação à soma do número de moles dos monômeros. A fase orgânica foi preparada dissolvendo-se o iniciador na mistura de monômeros, estireno (Sty) e divinilbenzeno (DVB), à temperatura ambiente. Em seguida, foi adicionado o diluente (mistura de tolueno e n-heptano) e finalmente a solução foi homogeneizada. A razão entre a fase aquosa e a fase orgânica foi mantida em 4/1 (v/v). Inicialmente, adicionou-se ao balão reacional a fase aquosa sob agitação mecânica e em seguida foi adicionada a fase orgânica. A fase orgânica foi dispersa na fase aquosa durante 15 minutos por meio de agitação, em seguida, a mistura reacional, sob agitação, foi aquecida a $70^{\circ} \mathrm{C}$ em banho termostatizado. Após 30 horas de reação, as pérolas de suporte Sty/DVB resultantes foram separadas por filtração a pressão reduzida (Figura 1).

As pérolas de Sty/DVB foram transferidas para um bécher ao qual foi adicionada água deionizada em quantidade aproximadamente duas a três vezes o volume das pérolas. A mistura foi agitada durante 30 minutos sob aquecimento $\left(\cong 50^{\circ} \mathrm{C}\right)$ e em seguida fil-

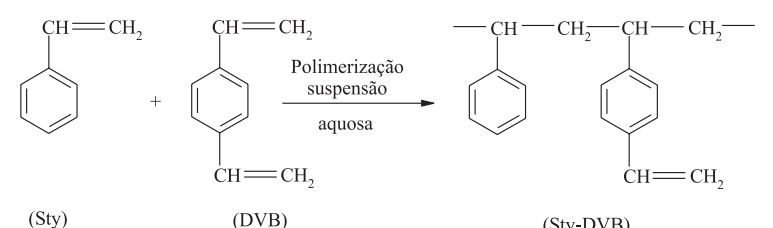

Figura 1. Representação esquemática da reação de copolimerização de estireno com divinilbenzeno 
trada a quente em funil de Buchner sob pressão reduzida. Esse tratamento foi repetido até que o filtrado se apresentasse límpido. O mesmo procedimento foi feito utilizando etanol até que o filtrado fosse solúvel em água ${ }^{[10]}$. As pérolas do suporte foram então peneiradas em peneirador hidráulico utilizando-se peneiras de 25, 45, 80, 100, 140 e 200 mesh. As pérolas separadas na faixa granulométrica de 45-80 mesh foram lavadas com metanol e secas em estufa, a aproximadamente $60^{\circ} \mathrm{C}$, por 48 horas $^{[10,11]}$. As pérolas de Sty/DVB dessa faixa granulométrica foram utilizadas para os testes de caracterização e como suporte para a produção dos catalisadores sulfônicos .

O objetivo da lavagem com água foi eliminar os estabilizadores da suspensão. A lavagem com etanol teve a finalidade de eliminar os monômeros residuais e diluente dos poros do suporte. $\mathrm{O}$ tratamento com metanol, antes da secagem, visava a obtenção de estruturas com poros livres de impurezas.

\section{Sulfonação do suporte polimérico}

A reação de sulfonação das pérolas de suporte de Sty/DVB foi realizada em aparelhagem semelhante à utilizada na síntese dos suportes, sendo que o balão usado foi de $250 \mathrm{ml}$ de capacidade, pois o volume reacional empregado era menor. Foi usada uma velocidade de agitação suficiente apenas para a homogeneização do meio, de modo a evitar a quebra das pérolas de suportes durante a sulfonação.

Na reação de sulfonação foram utilizados cerca de $3 \mathrm{~g}$ de suporte seco, preparado previamente, com granulometria de 45-80 mesh. O suporte foi inicialmente inchado em 1,2-dicloroetano ( $10 \mathrm{ml}$ de solvente para $1 \mathrm{~g}$ de suporte) durante 24 horas. Em seguida foi adicionado um excesso de ácido sulfúrico concentrado, agente sulfonante, utilizado na proporção $15 \mathrm{ml}$ para $1 \mathrm{~g}$ de suporte. A reação foi mantida durante 1 hora a $90^{\circ} \mathrm{C}$.

Após a sulfonação, a resina sulfonada foi vertida lentamente para um bécher de $250 \mathrm{ml}$ de capacidade contendo $50 \mathrm{ml}$ de água destilada. A mistura desse bécher foi transferida lentamente para outro bécher também contendo $50 \mathrm{ml}$ de água. Uma outra diluição foi realizada utilizando-se $1000 \mathrm{ml}$ de água. A diluição é feita aos poucos devido à alta concentração de ácido, que em contato com grande quantidade de água pode aumentar a pressão osmótica nos poros do catalisador ocasionando a quebra das pérolas.

\section{Caracterização dos materiais}

Os suportes e os catalisadores foram caracterizados pelos métodos descritos a seguir:

Densidade aparente - determinada utilizando uma modificação do método ASTM D1895 ${ }^{[12]}$.

Área específica e volume de poros - determinados por meio de dados de adsorção de nitrogênio a diferentes pressões relativas, na temperatura do nitrogênio líquido em equipamento ASAP (Micromeritics, Mod. 2010 - ASAP). A área específica foi determinada seguindo o método BET e o volume de poros segundo o método $\mathrm{BJH}^{[13,14]}$. No caso dos catalisadores, a área específica e o volume de poros somente foram determinados para as amostras cujos suportes precursores apresentaram um valor de área específica considerável, isto é, maior que $10 \mathrm{~m}^{2} / \mathrm{g}$.

Volume de poros - determinado por meio de medidas de retenção de água. Esse método se baseia na determinação do volume de água retido por massa de amostra após seu tratamento com metanol. Devido à superfície hidrofóbica dos suportes, a água não consegue penetrar em seus poros, tornando-se necessário reduzir a sua hidrofobicidade por meio de tratamento com metanol ${ }^{[15]}$. Para os catalisadores sulfônicos, essa determinação não foi realizada, pois eles apresentam caráter hidrofílico o que ocasionaria erros nas medidas. $\mathrm{O}$ total de água adsorvido seria o somatório do volume de água que penetra nos poros fixos e colapsados e a que incha os núcleos poliméricos, esta última resultante do caráter hidrofílico desses catalisadores.

Inchamento percentual em solventes - determinado por meio de retenção de solvente $\left(\mathrm{cm}^{3}\right.$ de solvente/grama de amostra) por centrifugação. No caso dos suportes, o inchamento foi realizado em dois tipos de solventes de características diferentes, tolueno (bom solvente) e n-heptano (mau solvente). Para os catalisadores, o inchamento percentual foi realizado em água e na mistura ácido acético/álcool n-butílico (razão molar 2/1). O inchamento percentual em água é um meio de avaliar a hidrofilicidade do catalisador.

Avaliação do aspecto morfológico - o aspecto morfológico do suporte e dos catalisadores sulfônicos imobilizados foi observado com microscópio óptico (Olympus, Mod. SZ10), equipado com câmera fotográfica, e com microscópio eletrônico de varredura (Digital Scanning Microscope DSM 960 ZEISS). 
Espectroscopia na região do infravermelho - os suportes e os catalisadores foram caracterizados por espectroscopia na região do infravermelho (PerkinElmer 1720X FT-IR). As amostras foram analisadas em pastilhas de $\mathrm{KBr}$ contendo aproximadamente $1 \%$ $\mathrm{p} / \mathrm{p}$ de amostra. Foi feito um total de 50 aquisições (scans) usando resolução de $4 \mathrm{~cm}^{-1}$, na faixa de frequiências de 4000 a $400 \mathrm{~cm}^{-1[16]}$.

Termogravimetria - cerca de $20 \mathrm{mg}$ de amostra foram colocados em cápsula de platina e esta foi aquecida em atmosfera de nitrogênio de $50^{\circ} \mathrm{C}$ a $850^{\circ} \mathrm{C}$ a uma velocidade de $10^{\circ} \mathrm{C} / \mathrm{min}$. Foi usado um analisador termogravimétrico Perkin-Elmer TGA-7.

Capacidade de troca catiônica e reciclagem dos catalisadores sulfônicos - o número de grupos capazes de trocar cátions é determinado por conversão dos grupos sulfônicos do catalisador para a forma ácida $\left(\mathrm{H}^{+}\right)$por meio do tratamento dos catalisadores com um excesso de ácido nítrico $\left(\mathrm{HNO}_{3}\right)$. Após o tratamento com ácido, o catalisador é lavado com água deionizada para remover o excesso de ácido e em seguida neutralizado com um excesso conhecido de solução padrão de hidróxido de sódio $(\mathrm{NaOH})$. Esse mesmo procedimento foi utilizado na reciclagem do catalisador sulfônico ${ }^{[17]}$.

Determinação da atividade catalítica em relação à esterificação do ácido acético com n-butanol - na reação de esterificação foi utilizado $1 \mathrm{~g}$ de resina sulfonada, ou catalisador, sendo adicionado em seguida o n-butanol (n-BuOH) e o ácido acético (HAc). Essa reação foi realizada em balão de três bocas de fundo redondo, de $250 \mathrm{ml}$ de capacidade, equipado com agitador mecânico e condensador de refluxo, a agitação empregada foi a suficiente para homogeneizar o meio. Um excesso de ácido acético foi utilizado para deslocar o equilíbrio em favor do éster. A mistura foi mantida sob refluxo durante 2 horas. Para todas as reações foi utilizada a razão molar HAc/ $\mathrm{n}$-BuOH igual a $2 / 1^{[18]}$. Foram determinados o rendimento da reação em gramas e a atividade catalítica em gramas de éster por meq de grupos sulfônicos por hora.

\section{Resultados e Discussão}

Neste trabalho foram utilizadas misturas de tolueno e n-heptano (diluente) como agentes formadores de poros das pérolas reticuladas de estirenodivinilbenzeno. Esses solventes foram escolhidos com
Tabela 1. Classificação dos solventes segundo a diferença entre os parâmetros de solubilidade em $\left(\mathrm{cal} / \mathrm{cm}^{3}\right)^{1 / 2}$ em relação a um polímero

\begin{tabular}{ll}
\hline Bom solvente & $0<\left|\delta_{\mathrm{P}}-\delta_{\mathrm{S}}\right|<0,5$ \\
\hline Solvente intermediário & $0,5<\left|\delta_{\mathrm{P}}-\delta_{\mathrm{S}}\right|<1,5$ \\
\hline Mau solvente & $1,5<\left|\delta_{\mathrm{P}}-\delta_{\mathrm{S}}\right|$ \\
\hline
\end{tabular}

$\delta_{\mathrm{P}}=$ parâmetros de solubilidade do polímero;

$\delta_{\mathrm{s}}=$ parâmetros de solubilidade do solvente

Tabela 2. Parâmetros de solubilidade do poliestireno e dos solventes da mistura diluente utilizada na síntese dos suportes ${ }^{[19]}$

\begin{tabular}{ccc}
\hline Solvente/Polímero & $\boldsymbol{\delta}\left[\left(\mathbf{c a l} / \mathbf{c m}^{3}\right)^{\mathbf{1 / 2}}\right]$ & $\left|\boldsymbol{\delta}_{\mathbf{P}}-\boldsymbol{\delta}_{\mathrm{S}}\right|\left(\mathbf{c a l} / \mathbf{c m}^{3}\right)^{\mathbf{1 / 2}}$ \\
\hline Poliestireno & 9,3 & \\
Tolueno & 9,0 & 0,3 \\
n-Heptano & 7,4 & 1,9 \\
\hline
\end{tabular}

base em critérios de solubilidade, ou de inchamento no caso de polímeros reticulados ${ }^{[19]}$.

Os solventes podem ser classificados, como regra geral, em bons, intermediários e maus solventes para um determinado polímero, segundo limites, não muito rígidos (Tabela 1 ).

Dentro desse critério, o tolueno é um bom solvente para o poliestireno, enquanto o n-heptano é um mau solvente. Os valores de parâmetro de solubilidade $(\delta)$ dos solventes e do polímero estão listados na Tabela 2. Quando são usadas misturas de solventes, o parâmetro de solubilidade da mistura $\left(\delta_{\mathrm{m}}\right)$ é uma média volumétrica daqueles dos solventes puros.

Além do critério do parâmetro de solubilidade, a análise da semelhança da estrutura química também deve ser considerada. O poliestireno é um polímero que contém anéis benzênicos em sua estrutura bem como o tolueno, enquanto que o n-heptano é um solvente alifático.

Se o sistema diluente dos monômeros é um bom agente de solvatação para o polímero em formação, as cadeias poliméricas ficam mais estendidas e a separação de fases, devido à insolubilidade do polímero reticulado, ocorre mais tarde e poros pequenos são produzidos. Quando o diluente é um mau solvente para as cadeias poliméricas em crescimento, estas ficam mais retraídas e a separação de fases ocorre mais cedo (em grau de conversão mais baixo) e poros maiores são gerados.

Com o objetivo de obter suportes poliméricos com diferentes características morfológicas foram sintetizadas quatro séries $(10,20,40$ e $60 \%$ de DVB) de 
Tabela 3. Condições gerais de síntese dos suportes poliméricos

\begin{tabular}{cccc}
\hline Suporte & $\begin{array}{c}\text { Tol/hep } \\
(\% \mathbf{v} / \mathbf{v})\end{array}$ & $\begin{array}{c}\text { DVB } \\
(\% \text { mol })\end{array}$ & $\begin{array}{c}\text { Sty } \\
(\% \text { mol })\end{array}$ \\
\hline SP-01 & & 10 & 90 \\
SP-02 & $70 / 30$ & 20 & 80 \\
SP-03 & & 40 & 60 \\
SP-04 & & 60 & 40 \\
\hline SP-05 & & 10 & 90 \\
SP-06 & $50 / 50$ & 40 & 80 \\
SP-07 & & 60 & 60 \\
SP-08 & & 10 & 90 \\
SP-09 & & 20 & 80 \\
SP-10 & & 40 & 60 \\
SP-11 & $30 / 70$ & 60 & 40 \\
SP-12 & & & 90 \\
\hline
\end{tabular}

Temperatura de polimerização $=70^{\circ} \mathrm{C}$; iniciador $\mathrm{r}=$ peróxido de benzoíla (1\% mol em relação aos monômeros); tol/hep: tolueno/ heptano; grau de diluição dos monômeros $=100 \%$ v/v copolímeros de estireno-divinilbenzeno. Esses foram preparados em condições reacionais em que se variou a composição do diluente (agente formador de poros) e se fixou o grau de diluição dos monômeros em 100 \% (v/v). A Tabela 3 mostra as condições gerais de síntese dos diferentes suportes poliméricos com as principais variáveis reacionais e, a Tabela 4 apresenta as características físicas destes, tais como: densidade aparente $\left(\mathrm{d}_{\mathrm{ap}}\right)$, área específica $(\mathrm{S})$, volume de poros fixos pelo método de retenção de água $\left(\mathrm{V}_{\mathrm{p}_{\mathrm{H} 2} \mathrm{O}}\right)$, volume de poros fixos por adsorção de nitrogênio pelo método BJH $\left(\mathrm{Vp}_{\mathrm{BJH}}\right)$, diâmetro de poros $(\overline{\mathrm{D}})$ e inchamento percentual em tolueno $\left(I_{\text {tol }}\right)$ e em heptano $\left(I_{\text {hep }}\right)$.

A Tabela 4 mostra que, para um mesmo teor de DVB, as características dos suportes, tanto no estado seco como no estado inchado, são afetadas pela variação da composição do diluente (tolueno/ heptano).

A maior ou menor afinidade da mistura diluente pelo copolímero em formação determina a ocorrência da separação de fases, durante o processo de polimerização, que leva à formação da porosidade no estado seco.

Tabela 4. Características físicas dos suportes poliméricos

\begin{tabular}{|c|c|c|c|c|c|c|c|c|c|}
\hline $\begin{array}{c}\text { DVB } \\
(\% \mathrm{~mol})\end{array}$ & Suporte & $\begin{array}{l}\text { Tol/hep } \\
(\% \text { v/v) }\end{array}$ & $\underset{\left(\mathrm{g} / \mathrm{cm}^{3}\right)}{\mathrm{d}_{\mathrm{ap}}}$ & $\begin{array}{c}\mathrm{S} \\
\left(\mathrm{m}^{2} / \mathrm{g}\right)\end{array}$ & $\begin{array}{c}V p_{\mathrm{BJH}} \\
\left(\mathrm{cm}^{3} / \mathrm{g}\right)\end{array}$ & $\begin{array}{c}V p_{\mathbf{H}_{2} \mathbf{o}} \\
\left(\mathrm{cm}^{3} / \mathbf{g}\right)\end{array}$ & $\underset{(\stackrel{\mathbf{D}}{)})}{(\AA)}$ & $\begin{array}{l}I_{\text {tol }} \\
(\%)\end{array}$ & $\begin{array}{l}I_{\text {hep }} \\
(\%)\end{array}$ \\
\hline \multirow{3}{*}{10} & SP-01 & $70 / 30$ & 0,63 & 0,29 & nd & 0,040 & nd & 263 & 4 \\
\hline & SP-09 & $50 / 50$ & 0,64 & 0,72 & nd & 0,120 & nd & 256 & 2 \\
\hline & SP-05 & $30 / 70$ & 0,58 & 0,39 & nd & 0,03 & nd & 231 & 0 \\
\hline \multirow{3}{*}{20} & SP-02 & $70 / 30$ & 0,65 & 0,47 & nd & 0,010 & nd & 192 & 0 \\
\hline & SP-10 & $50 / 50$ & 0,66 & 0,39 & nd & 0,100 & nd & 156 & 5 \\
\hline & SP-06 & $30 / 70$ & 0,59 & 0,30 & nd & 0,020 & nd & 148 & 19 \\
\hline \multirow{3}{*}{40} & SP-03 & $70 / 30$ & 0,62 & 0,64 & nd & 0,060 & nd & 92 & 54 \\
\hline & SP-11 & $50 / 50$ & 0,61 & 20 & 0,053 & 0,180 & 92 & 90 & 74 \\
\hline & SP-07 & $30 / 70$ & 0,53 & 93 & 0,224 & 0,230 & 85 & 66 & 81 \\
\hline \multirow{3}{*}{60} & SP-04 & $70 / 30$ & 0,62 & 10 & 0,010 & 0,220 & 62 & 64 & 91 \\
\hline & SP-12 & $50 / 50$ & 0,55 & 237 & 0,246 & 0,480 & 59 & 69 & 60 \\
\hline & SP-08 & $30 / 70$ & 0,45 & 304 & 0,472 & 0,610 & 83 & 39 & 39 \\
\hline
\end{tabular}

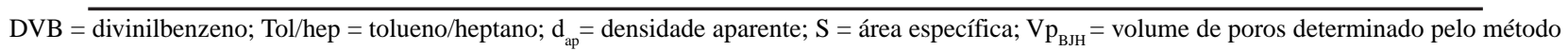

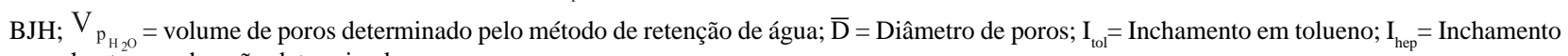
em n-heptano; nd = não determinado 


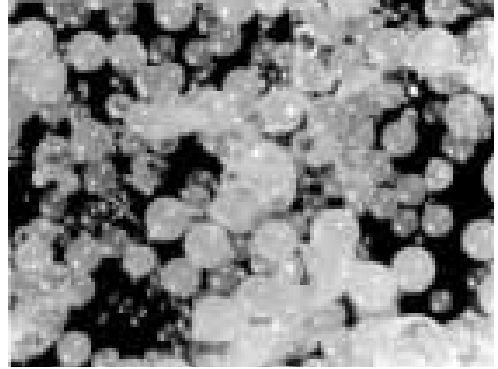

(a) SP-05

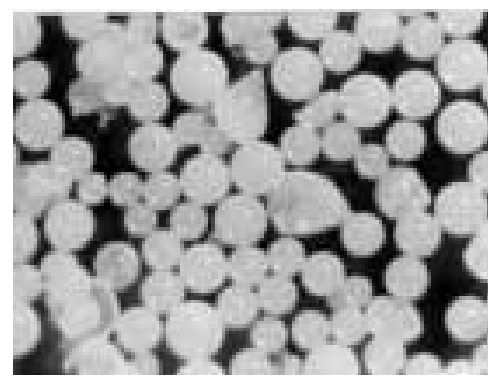

(c) SP-07

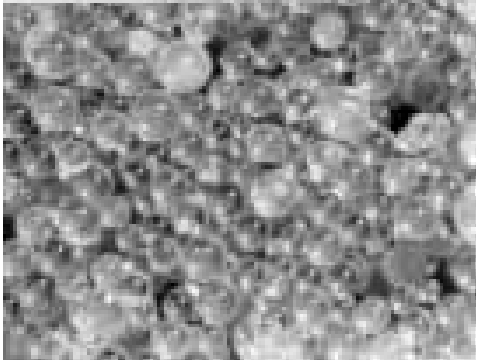

(b) SP-06

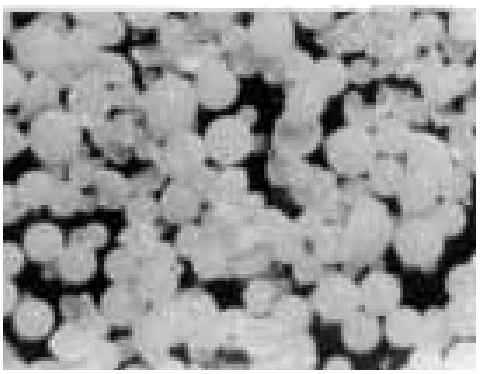

(d) SP-08

Figura 2. Micrografias de microscopia ótica de suportes poliméricos preparados usando como diluente mistura tolueno/heptano em composição 30/70 com 10 (a), 20 (b), 40 (c) e $60 \%$ (d) de DVB (aumento 18X)

Os suportes obtidos com baixos teores de DVB (10 e $20 \%$ ) não apresentaram porosidade no estado seco, mesmo em presença de misturas diluente ricas em n-heptano (mau solvente). Pode-se observar (Tabela 4) que a densidade aparente, a área específica e $\mathrm{o}$ volume de poros $\left(\mathrm{Vp}_{\mathrm{H}_{2} \mathrm{O}}\right)$, determinado pela retenção de água, foram pouco afetados pela composição da mistura diluente. Foram obtidos suportes com estruturas morfológicas do tipo gel, confirmadas pelo seu aspecto óptico transparente, que denota uma estrutura homogênea, apresentando uma só fase. O aspecto visual desses suportes, preparados com composição tol/hep 30/70 e com 10 e $20 \%$ de DVB, é mostrado na Figura 2 (a) e (b), respectivamente, por meio de micrografias de microscopia ótica.

Os suportes preparados com 40 e $60 \%$ de DVB apresentaram um decréscimo na densidade aparente, isto é, os suportes tornaram-se mais porosos, à medida que o teor de n-heptano na mistura diluente aumentou. $\mathrm{O}$ aumento da porosidade é confirmado pelos valores de volume de poros fixos $\left(\mathrm{Vp}_{\mathrm{H}_{2} \mathrm{O}}\right)$ e de área específica. Esses valores aumentaram com o acréscimo do teor de DVB, e para um mesmo teor de DVB, aumentaram com o aumento do teor do mau solvente (n-heptano) na mistura diluente. A área específica foi a característica mais afetada pelo aumento combinado do teor de DVB do mau solvente na mistura diluente. Isso se deve ao efeito acentuado que essas variáveis combinadas têm sobre o processo de separação de fases, que leva à formação dos poros fixos ${ }^{[20]}$.

Esses resultados estão de acordo com o mecanismo de formação da estrutura porosa, isto é, à medida que o teor de DVB aumenta e o diluente se torna

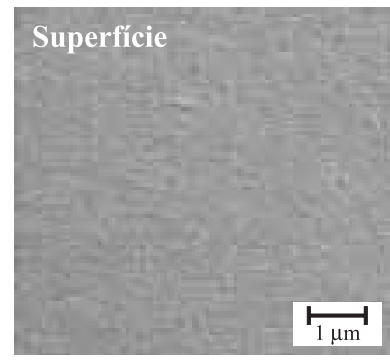

(a) $\mathrm{SP}-04$

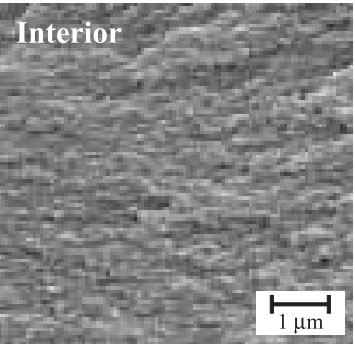

(c) $\mathrm{SP}-04$

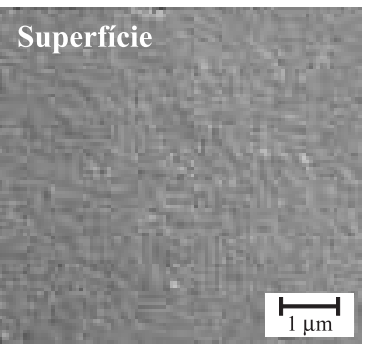

(b) SP-08

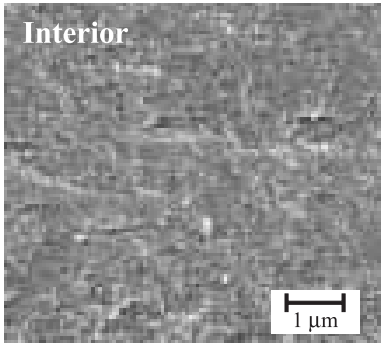

(d) SP-08
Figura 3. Micrografias de microscopia eletrônica de varredura dos suportes poliméricos $\mathrm{SP}-04$ (a) e (c) preparado com $60 \%$ de DVB e com mistura diluente tol $/$ hep $=70 / 30$ e SP-08 (b) e (d) preparado com $60 \%$ de DVB e com mistura tol/hep $=30 / 70$ 
menos solvatante, a porosidade do material aumenta. Os suportes assim produzidos apresentam porosidade no estado seco. $\mathrm{O}$ aspecto visual desses suportes (aparência opaca) preparados com composição tol/hep $30 / 70$ com 40 e $60 \%$ de DVB pode ser observado por meio das micrografias de microscopia ótica apresentadas na Figura 2 (c) e (d), respectivamente. A porosidade da superfície e do interior das pérolas do suporte preparados com $60 \%$ de DVB pode ser visualizada nas micrografias de microscopia eletrônica de varredura apresentadas na Figura $3^{[14]}$.

Estruturas poliméricas do tipo gel, de um modo geral, apresentam uma maior capacidade de retenção de um bom solvente do que as estruturas macroporosas. Isso se deve à maior elasticidade da estrutura do tipo gel, devido ao menor grau de reticulação das cadeias poliméricas.

$\mathrm{O}$ aumento do teor do mau solvente na síntese do suporte tende a produzir estruturas reticuladas mais emaranhadas, o que pode levar a um decréscimo da sua capacidade de inchamento ${ }^{[20]}$.

Com o aumento do teor de DVB, as estruturas tornam-se mais rígidas, ocorrendo um decréscimo nos valores de inchamento dos suportes em tolueno. As estruturas macroporasas são constituídas por duas fases, fase poro e fase gel, e a retenção de tolueno (bom solvente) tende a ser mais alta do que a retenção de n-heptano (mau solvente). Quanto mais predominante for a fase poro sobre a fase gel, mais próximos serão os valores inchamento no bom solvente e no mau solvente.

Os suportes obtidos com $10 \%$ de DVB em diferentes razões do diluente tol/hep apresentaram apenas uma pequena variação nos valores de inchamento, tanto no bom (tolueno) como no mau (heptano) solvente (Tabela 4). Esse resultado pode ser atribuído ao fato desse suporte ter uma capacidade de inchamento muito alta em tolueno e muito baixa em heptano.

Os catalisadores sulfônicos imobilizados nos suportes poliméricos foram preparados em presença de um bom solvente, 1,2-dicloroetano, para que a reação de sulfonação ocorresse na superfície do interior dos poros fixos, na superfície externa do suporte, bem como na matriz polimérica inchada. Foram correlacionadas as características físicas do catalisador sulfônico no estado seco e as do suporte que lhe deu origem (Tabela 4). A Tabela 5 mostra as características físicas dos catalisadores sulfônicos produzidos a partir dos supor-
Tabela 5. Características físicas dos catalisadores sulfônicos

\begin{tabular}{|c|c|c|c|c|}
\hline $\begin{array}{c}\text { DVB } \\
(\% \mathrm{~mol})\end{array}$ & Catalisador & $\begin{array}{l}\text { Tol/hep } \\
(\% \text { v/v) }\end{array}$ & $\underset{\left(\mathrm{g} / \mathrm{cm}^{3}\right)}{\mathbf{d}_{\mathrm{ap}}}$ & $\begin{array}{c}\mathbf{S} \\
\left(\mathrm{m}^{2} / \mathrm{g}\right)\end{array}$ \\
\hline \multirow{3}{*}{10} & CP-01 & $70 / 30$ & 0,78 & nd \\
\hline & CP-09 & $50 / 50$ & 0,73 & nd \\
\hline & CP-05 & $30 / 70$ & 0,74 & nd \\
\hline \multirow{3}{*}{20} & CP-02 & $70 / 30$ & 0,78 & nd \\
\hline & CP-10 & $50 / 50$ & 0,75 & nd \\
\hline & CP-06 & $30 / 70$ & 0,75 & nd \\
\hline \multirow{3}{*}{40} & $\mathrm{CP}-03$ & $70 / 30$ & 0,76 & nd \\
\hline & CP-11 & $50 / 50$ & 0,73 & 7 \\
\hline & CP-07 & $30 / 70$ & 0,72 & 31 \\
\hline \multirow{3}{*}{60} & CP-04 & $70 / 30$ & 0,68 & 12 \\
\hline & CP-12 & $50 / 50$ & 0,66 & 83 \\
\hline & CP-08 & $30 / 70$ & 0,63 & 111 \\
\hline
\end{tabular}

DVB = divinilbenzeno; Tol/hep = tolueno/heptano utilizado na síntese dos suportes; $\mathrm{d}_{\mathrm{ap}}=$ densidade aparente; $\mathrm{S}$ = área específica; $\mathrm{nd}=$ não determinadas, pois as áreas dos suportes correspondentes apresentaram valores muito baixos

tes sintetizados com 10, 20, 40 e $60 \%$ de DVB em diferentes composições do diluente (tol/ hep).

De um modo geral, a densidade aparente dos catalisadores aumentou em relação àquelas dos suportes precursores, devido à introdução de grupos funcionais contendo átomos mais pesados do que o carbono, no caso o enxofre, e à fixação destes no interior dos poros com diminuição da porosidade; conseqüentemente este fato leva a um decréscimo da área específica (Tabela 5).

Comparando os resultados de área específica, de um modo geral, os valores determinados para os catalisadores sulfônicos são inferiores aos dos suportes precursores. Por exemplo os catalisadores sulfônicos CP-8 $\left(111 \mathrm{~m}^{2} / \mathrm{g}\right)$, CP-11 $\left(7 \mathrm{~m}^{2} / \mathrm{g}\right)$ e CP-12 $\left(83 \mathrm{~m}^{2} / \mathrm{g}\right)$ possuem áreas específicas inferiores aos suportes correspondentes, SP-8 $\left(304 \mathrm{~m}^{2} / \mathrm{g}\right), \mathrm{SP}-11\left(20 \mathrm{~m}^{2} / \mathrm{g}\right)$ e SP-12 $\left(237 \mathrm{~m}^{2} / \mathrm{g}\right)$. Somente para o catalisador CP-04 $\left(12 \mathrm{~m}^{2} / \mathrm{g}\right)$ foi observado um aumento no valor da área específica em relação ao suporte SP-04 $\left(10 \mathrm{~m}^{2} / \mathrm{g}\right)$. Esse aumento pode ser atribuído à quebra das pérolas, resultante da tensão gerada pela entrada do grupo sulfônico na 

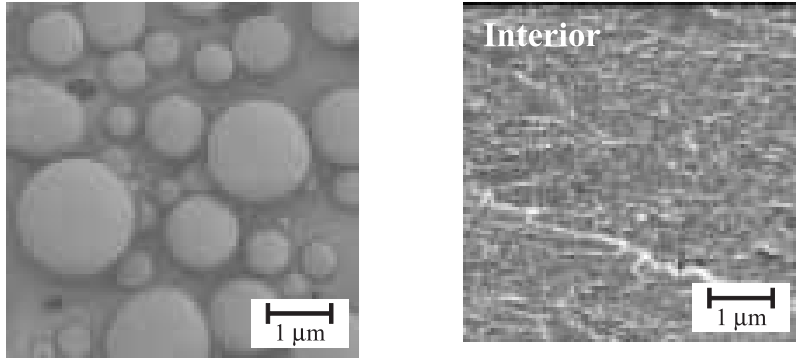

(a) suporte polimérico SP-04
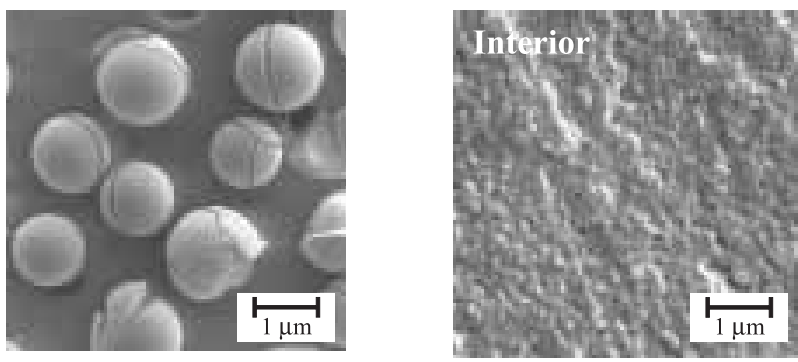

(b) catalisador sulfônico CP-04

Figura 4. Micrografias de microscopia eletrônica de varredura: (a) suporte SP-04 preparado com $60 \%$ de DVB e com mistura diluente tol/ hep $=70 / 30$ e (b) do catalisador CP-04 correspondente

estrutura mesoporosa do suporte. Esse efeito pode ser observado na Figura 4 (a) e (b) que mostra micrografias de microscopia eletrônica de varredura do suporte e catalisador, respectivamente. Pode-se observar claramente rachaduras na superfície das pérolas do catalisador CP-04 bem como maior rugosidade da sua superfície interna em relação à do suporte SP-04.

Os suportes preparados com 10 e $60 \%$ de DVB e os respectivos catalisadores foram caracterizados por espectroscopia na região do infravermelho - FT-IR (Figura 5). Foi observado que, para os catalisadores, os espectros não apresentaram uma boa resolução, devido à higroscopicidade que o grupo $-\mathrm{SO}_{3} \mathrm{H}$ confere ao material. Foi observada uma banda na faixa de frequiências de $1230 \mathrm{~cm}^{-1}$ a $1120 \mathrm{~cm}^{-1}$, característica do grupo sulfonato. Ácidos sulfônicos, quando em sua forma anidra, absorvem em duas regiões estreitas de frequiências: $1350-1342 \mathrm{~cm}^{-1} \mathrm{e}$ em $1165-1150 \mathrm{~cm}^{-1}$. Porém, em ausência de tratamento especial de secagem das amostras e manipulação em atmosfera anidra, os grupos sulfônicos se hidratam rapidamente formando sais sulfonatos de hidrônio, que absorvem na faixa de freqüências de $1230 \mathrm{~cm}^{-1}$ a $1120 \mathrm{~cm}^{-1[21]}$.

A estabilidade térmica dos suportes e dos catalisadores foi avaliada por termogravimetria. Os resultados dessa análise mostraram que os suportes apresentam apenas um estágio de degradação, que

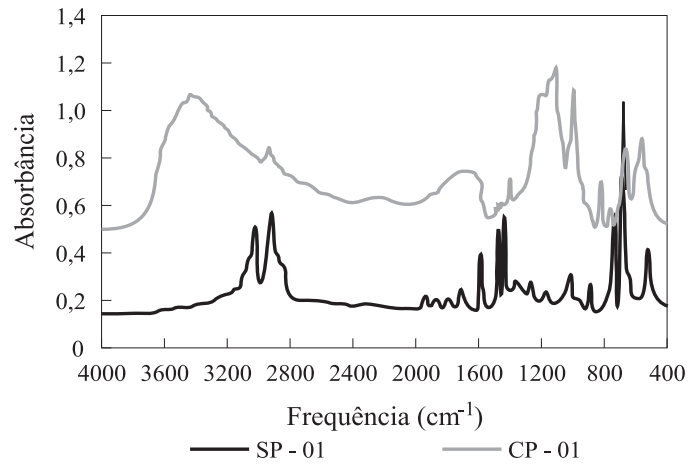

(a) SP-01 e CP-01

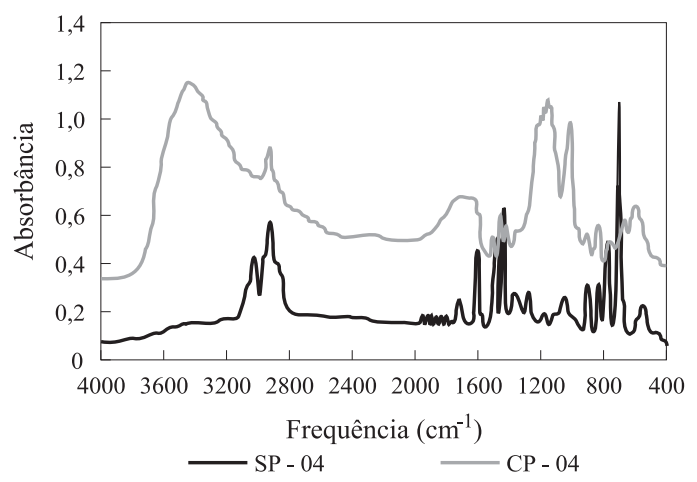

(b) $\mathrm{SP}-04$ e CP-04

Figura 5. Espectros de FT-IR dos suportes e dos catalisadores: (a) suporte SP-01 (tol/hep $=70 / 30,10 \%$ de DVB) e do catalisador CP-01 correspondente e (b) suporte SP-04 (tol/hep $=70 / 30,60 \%$ de DVB) e do catalisador $\mathrm{CP}-04$ correspondente

variou de acordo com o grau de reticulação: $10 \%$ de DVB (SP-01 $-\mathrm{T}=246^{\circ} \mathrm{C}$ ) e $60 \%$ de DVB (SP-11 $\mathrm{T}=283^{\circ} \mathrm{C}$ ), Figura 6 (a) e (b), respectivamente. $\mathrm{O}$ suporte mais reticulado (SP-11) tem menor probabilidade de sofrer degradação, pois necessita que um número maior de ligações sejam quebradas para que ocorra a perda de massa e assim o início da degradação ocorre em temperaturas mais elevadas $\left(\mathrm{T}=283^{\circ} \mathrm{C}\right)$.

As curvas de TG/DTG de quatro catalisadores sulfônicos com diferentes características morfológicas mostraram que estes apresentam perda de massa em três zonas de temperatura distintas (Figura 7).

A primeira zona de perda de massa, entre 50 e $200^{\circ} \mathrm{C}$, deve-se principalmente à dessolvatação de água, uma vez que os catalisadores são higroscópicos. Nessa primeira etapa de perda de massa foi observada uma elevação da temperatura final em função do teor de DVB no suporte polimérico. A primeira perda de massa vai até $217^{\circ} \mathrm{C}$, para o catalisador CP-01 (Figura 7 a) até $203^{\circ} \mathrm{C}$, para o catalisador CP-02 (Figura 7 b) até $200^{\circ} \mathrm{C}$ e para o catalisador CP-03 (Figu- 


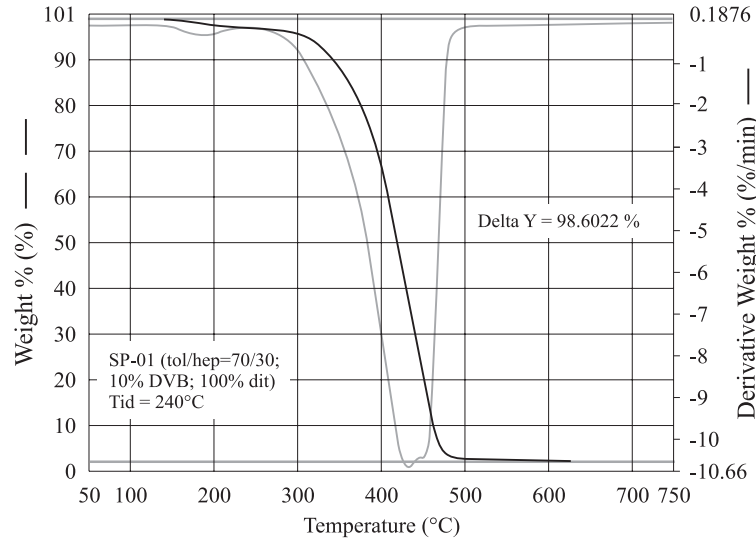

(a) SP-01

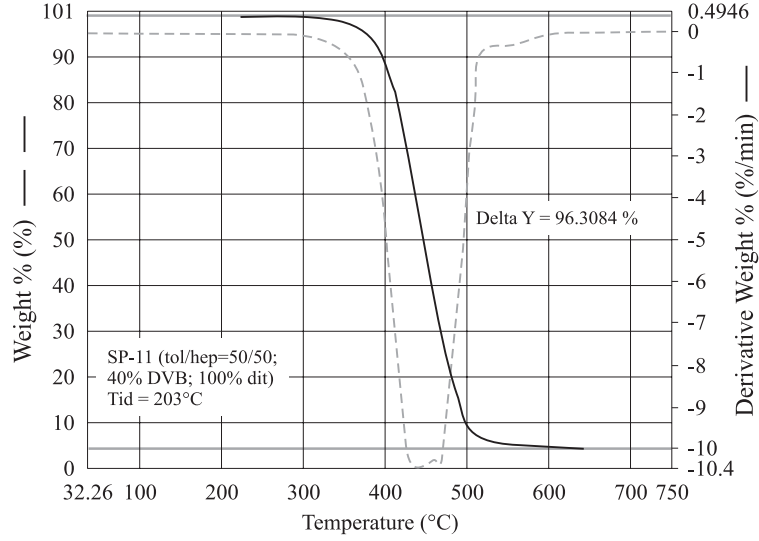

(b) SP-11

Figura 6. Curvas de TG e DTG dos suportes poliméricos: (a) SP-01, tol/hep = 70/30; 10 \% DVB e (b) SP-11, tol/hep= 50/50; 40 \% DVB (taxa de aquecimento $=10^{\circ} \mathrm{C} / \mathrm{min}$, atmosfera de nitrogênio)

ra 7 c) até $194^{\circ} \mathrm{C}$ para o catalisador $\mathrm{CP}-04$ preparados respectivamente com suportes poliméricos contendo 10, 2040 e $60 \%$ de DVB. Esse comportamento

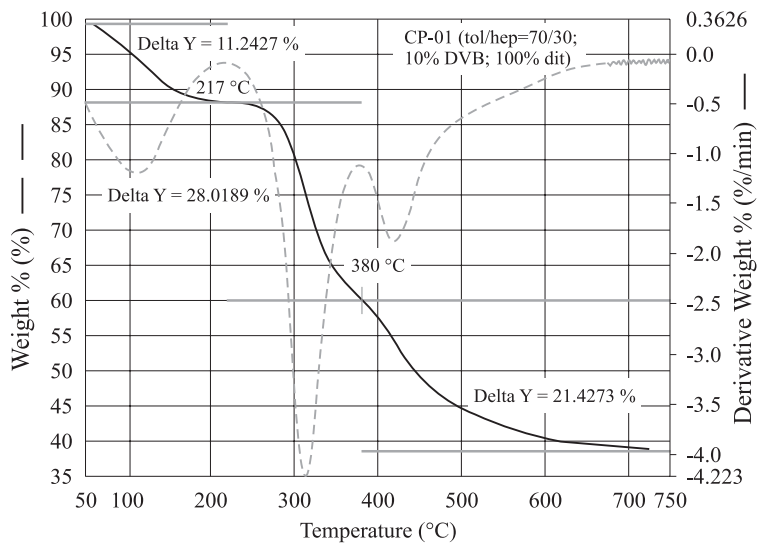

(a) CP-01

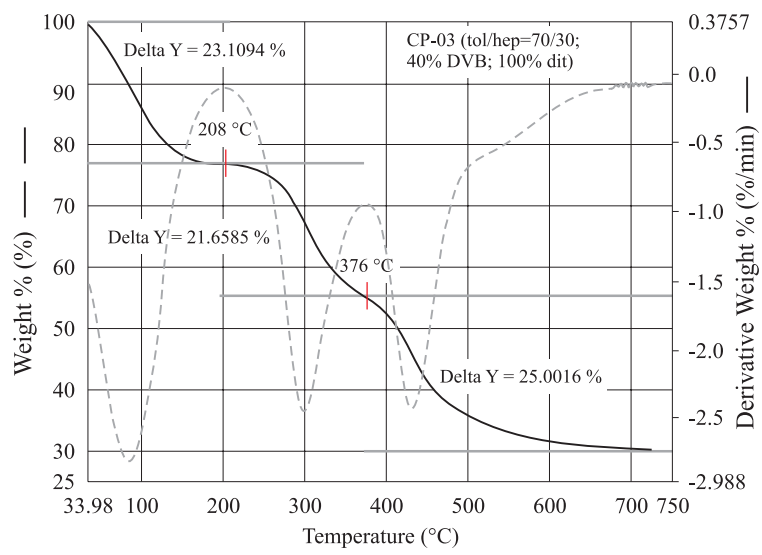

(c) $\mathrm{CP}-03$ pode ser atribuído ao volume de poros fixos, quanto maior for esse volume mais facilmente a água sairá da estrutura polimérica.

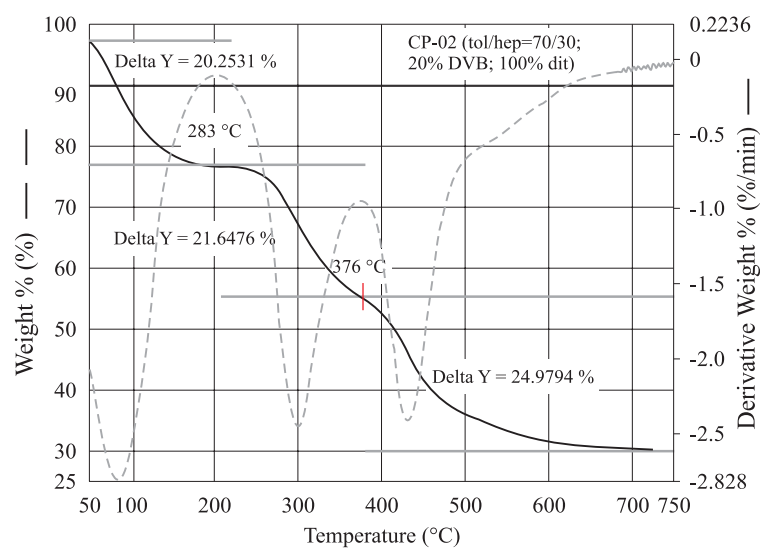

(b) $\mathrm{CP}-02$

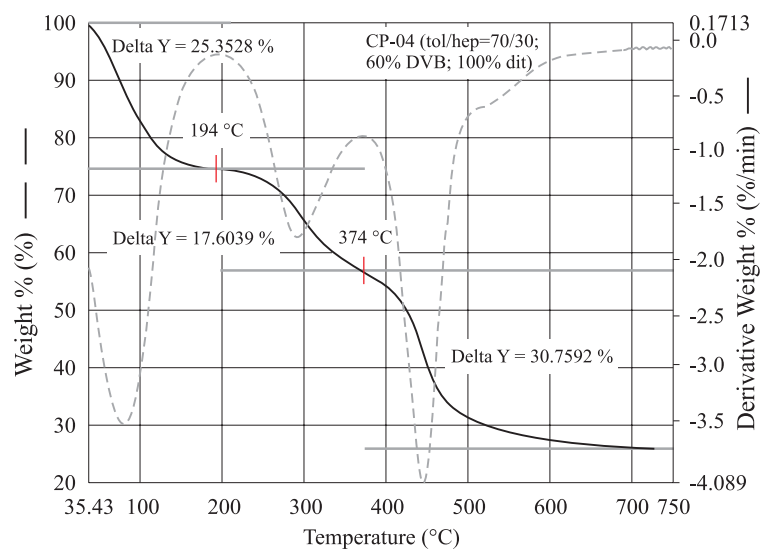

(d) $\mathrm{CP}-04$

Figura 7: Curvas de TG e DTG dos catalisadores sulfônicos: (a) CP-01, (b) CP-02, (c) CP-03 e (d) CP-04, produzidos a partir de suportes poliméricos preparados com mistura tol/hep $=70 / 30$ contendo, respectivamente, 10, 20, 40 e $60 \% \mathrm{DVB}$ (taxa de aquecimento $10^{\circ} \mathrm{C} / \mathrm{mim}$, atmosfera de nitrogênio) 
A segunda zona de perda de massa, entre 200 e $400^{\circ} \mathrm{C}$, é atribuída à dessulfonação térmica do catalisador e a terceira, entre 340 e $500^{\circ} \mathrm{C}$, é devido à degradação do suporte ou da matriz polimérica ${ }^{[22-24]}$.

Em atmosfera de nitrogênio, o início da dessulfonação, para todos os catalisadores, ocorreu em temperatura acima de $200^{\circ} \mathrm{C}$. À medida que o teor de DVB da estrutura do suporte do catalisador aumentou, houve um decréscimo na temperatura em que a velocidade de degradação é máxima. Esse efeito pode ser atribuído ao maior impedimento estérico dos suportes mais reticulados (fase gel), que seriam mais beneficiados pelo alívio de tensão causado pela saída dos volumosos grupos sulfônicos.

Os catalisadores também foram caracterizados em relação ao inchamento percentual em água e na mistura ácido acético/n-butanol (HAc/n-butanol - relação molar 2/1). De um modo geral, os valores de inchamento percentual diminuíram com o aumento do teor de DVB utilizado na síntese dos suportes precursores. O maior grau de reticulação diminui a elasticidade das cadeias poliméricas, diminuindo assim a capacidade de inchamento do suporte. Foi observado também que o aumento do teor de mau solvente (n-heptano) na mistura diluente favoreceu a diminuição do inchamento, devido à mais alta extensão de separação de fases, ocorrida durante o processo de síntese da rede polimérica, resultando assim em uma estrutura mais emaranhada, com menor capacidade de inchamento ${ }^{[20]}$.

Os valores de inchamento percentual em água foram visivelmente maiores que os de inchamento na mistura $\mathrm{HAc} / \mathrm{n}-\mathrm{BuOH}$. Isso se deve à mais alta afinidade do grupo sulfônico fixado no suporte pela água do que pela mistura $\mathrm{HAc} / \mathrm{n}-\mathrm{BuOH}$.

A Tabela 6 mostra os resultados de inchamento percentual, capacidade de troca catiônica e atividade catalítica dos catalisadores sulfônicos imobilizados em polímero.

A capacidade de troca catiônica dá uma medida do teor de grupos sulfônicos acessíveis em meio aquo$\mathrm{so}^{[25]}$. A quantidade de íons retidos pelo catalisador depende da afinidade do íon pelo catalisador e do grau de inchamento do mesmo.

Tabela 6: Características de inchamento percentual, capacidade de troca catiônica e atividade catalítica de catalisadores sulfônicos produzidos a partir dos suportes sintetizados com 10, 20, 40 e $60 \%$ de DVB em diferentes composições do diluente (tol/ hep)

\begin{tabular}{|c|c|c|c|c|c|c|}
\hline $\begin{array}{c}\text { DVB } \\
(\% \mathrm{~mol})\end{array}$ & Catalisadores & $\begin{array}{l}\text { Tol/hep } \\
\text { (\% v/v) }\end{array}$ & $\begin{array}{l}\mathbf{I}_{\mathrm{H}} \mathrm{o} \\
(\%)\end{array}$ & $\begin{array}{c}\text { Capacidade de } \\
\text { troca catiônica } \\
\left(\mathrm{meq}_{\mathrm{SO} \mathrm{H}} / \mathrm{g}\right)\end{array}$ & $\begin{array}{c}\mathbf{I}_{(\mathrm{HAc}-\mathrm{n}-\mathrm{BuOH})} \\
(\%)\end{array}$ & $\begin{array}{l}\text { Atividade catalítica } \\
\qquad\left(\mathrm{g}_{\text {éster }} / \mathbf{m e q}_{\mathrm{SO}_{\mathrm{H}}} \mathrm{h}\right)\end{array}$ \\
\hline & CP-01 & $70 / 30$ & 265 & 9,94 & 101 & 1,83 \\
\hline \multirow[t]{3}{*}{10} & CP-09 & $50 / 50$ & 280 & 9,47 & 74 & 1,88 \\
\hline & CP-05 & $30 / 70$ & 215 & 10,30 & 63 & 1,94 \\
\hline & CP-02 & $70 / 30$ & 147 & 8,45 & 52 & 2,19 \\
\hline \multirow[t]{3}{*}{20} & CP-10 & $50 / 50$ & 126 & 7,50 & 38 & 2,34 \\
\hline & CP-06 & $30 / 70$ & 107 & 8,20 & 80 & 2,45 \\
\hline & CP-03 & $70 / 30$ & 105 & 6,34 & 64 & 2,40 \\
\hline \multirow[t]{3}{*}{40} & $\mathrm{CP}-11$ & $50 / 50$ & 86 & 7,17 & 69 & 2,78 \\
\hline & CP-07 & $30 / 70$ & 69 & 7,20 & 24 & 2.45 \\
\hline & CP-04 & $70 / 30$ & 71 & 6,82 & 40 & 2,31 \\
\hline \multirow[t]{2}{*}{60} & $\mathrm{CP}-12$ & $50 / 50$ & 67 & 5,81 & 30 & 3,09 \\
\hline & CP-08 & $30 / 70$ & 51 & 5,80 & 22 & 2,91 \\
\hline
\end{tabular}

DVB = divinilbenzeno; Tol/hep = razão volumétrica tolueno/heptano utilizada na síntese dos suportes;

$\mathrm{I}_{\mathrm{H}_{2} \mathrm{O}}=$ Inchamento em água;

$\mathrm{I}_{(\mathrm{HAc}-\mathrm{n}-\mathrm{BuOH})}^{\mathrm{H}_{2} \mathrm{O}}=$ Inchamento na mistura HAc-n-BuOH 
Os valores de capacidade de troca catiônica mais altos foram atribuídos aos catalisadores que apresentaram os maiores valores de inchamento em água, ou seja, aqueles preparados com suportes poliméricos com os mais baixos teores de DVB (10 e $20 \%$ ). Esse comportamento pode ser atribuído à maior facilidade de acesso dos reagentes aos grupos sulfônicos situados no interior da estrutura polimérica do tipo gel (Tabela 6). Estruturas mais reticuladas (menos elásticas) podem impedir o acesso a certos grupos sulfônicos, dificultando a troca iônica, levando dessa forma a um decréscimo nos valores de capacidade de troca.

Os valores de atividade catalítica na reação de esterificação do ácido acético com n-butanol aumentaram à medida que se aumentou o teor de DVB. Foi observado que para o mesmo teor de DVB, o aumento do teor do mau solvente favoreceu um aumento nos valores de atividade catalítica. Dentre esses resultados, foi observado que, os catalisadores sulfônicos preparados com os suportes com teores de DVB de 40 e $60 \%$ e composição diluente tol/ hep de 50/50 apresentaram um comportamento diferenciado. Esses catalisadores apresentaram os mais altos valores de atividade catalítica.

A Tabela 7 mostra os resultados de reciclagem para dois catalisadores com diferentes morfologias: CP-05 (tipo gel) e CP-08 (mesoporoso). O catalisador mesoporoso (CP-08) apresentou valores mais altos de atividade que o CP-05.

Foram realizadas quatro reciclagens (ciclos) com esses catalisadores, e ao final de cada reação de esterificação o catalisador era regenerado com ácido nítrico, seguindo a mesma metodologia empregada na determinação da capacidade de troca catiônica. Os resultados obtidos mostraram que após a reciclagem ambos os catalisadores (CP-05 e

Tabela 7: Influência da reciclagem na atividade dos catalisadores poliméricos CP-05 e CP-08

\begin{tabular}{ccc}
\hline & \multicolumn{2}{c}{ Catalisador } \\
\cline { 2 - 3 } $\begin{array}{c}\text { Número } \\
\text { de ciclos }\end{array}$ & $\mathbf{C P - 0 5}$ & $\mathbf{C P - 0 8}$ \\
\cline { 2 - 3 } & \multicolumn{2}{c}{$\begin{array}{c}\text { Atividade Catalítica } \\
\left(\mathbf{g}_{\text {éster }} / \mathbf{m e q}_{\mathbf{S O}_{3} \mathbf{H}} \mathbf{h}\right)\end{array}$} \\
\hline Catalisador novo & 1,94 & 2,91 \\
1 & 2,91 & 4,82 \\
2 & 2,91 & 4,82 \\
3 & 3,01 & 5,00 \\
4 & 3,10 & 5,34 \\
\hline
\end{tabular}

CP-08) apresentaram atividades superiores ao catalisador não-reciclado (novo). Esse aumento pode ser atribuído ao tratamento ácido que os catalisadores sofreram na reciclagem, sugerindo que grande parte dos grupos sulfônicos estariam na forma de sulfonato de hidrônio no catalisador novo, sendo recuperados na forma de $\mathrm{SO}_{3} \mathrm{H}$, após tratamento com $\mathrm{HNO}_{3}$.

\section{Conclusões}

Para os suportes sintetizados usando como diluente a mistura tol/hep na composição 70/30, o aumento do teor de DVB praticamente não afetou os valores de densidade aparente. Nas séries em que se empregou a composição tol/hep 30/70 e 50/50, o aumento do teor de DVB diminuiu os valores de densidade aparente, indicando um aumento na porosidade. Somente com a combinação de altos teores de DVB e de mau solvente na mistura diluente foi possível obter estruturas porosas com altos valores de área específica e de volume de poros.

Após a reação de sulfonação do suporte, os catalisadores sulfônicos imobilizados em polímero, apresentaram um valor mais elevado de densidade aparente, em relação aos suportes precursores, devido à introdução de grupamentos com átomos mais pesados. Essa variação da densidade foi confirmanda pelos valores de área específica dos catalisadores, que diminuíram em relação aos suportes precursores. Somente para o catalisador CP-04 a área específica aumentou, em relação ao suporte precursor, devido à quebra das pérolas do suporte.

Ocorreu uma diminuição no inchamento dos catalisadores em solventes com o aumento do teor de DVB utilizado na síntese dos suportes poliméricos;

Os valores de capacidade de troca catiônica mais elevados foram obtidos para os catalisadores preparados com suportes com os mais baixos teores de DVB $(10$ e $20 \%)$.

De um modo geral, os catalisadores que apresentaram os valores maiores de atividade catalítica, foram aqueles que apresentaram os valores mais altos de porosidade, ou seja, aqueles preparados com suportes contendo 40 e $60 \%$ de DVB.

$\mathrm{O}$ tratamento dos catalisadores com $\mathrm{HNO}_{3}$, durante o processo de regeneração, provocou um aumento da atividade catalítica destes, quando comparados aos catalisadores novos (não reciclados). 


\section{Agradecimentos}

Os autores agradecem ao CNPq, PADCT/CNPq, FAPERJ (E-26/150846/99), Nitriflex, CENPES/ Petrobrás e ao Departamento de Ciência dos Materiais e Metalurgia da PUC-Rio.

\section{Referências Bibliográficas}

1. Siqueira, M.I.N. - "Síntese e caracterização de resinas de troca aniônica à base de 2-vinilpiridina $e$ divinilbenzeno", Tese de Mestrado, Universidade Federal do Rio de Janeiro, Brasil (1989).

2. Beauvais, R.A. \& Alexandratos, S.D. - React. Funct. Polym., 36, p. 113 (1998).

3. Struck, M. \& Widdecke, H. - Ang. Makromol. Chem., 235, p. 131 (1996).

4. Luca, C., Neagu, V., Simionescu, B.C., Rabia, I., Zerouk, J. \& Bencheikh,Z. - React. Funct. Polym., 36, p. 79 (1998).

5. Malshe, V.C. \& Sujatha, E.S. - React. Polym., 35, p. 159 (1997).

6. Sharma, M.N. - React. Polym., 26, p. 3 (1995).

7. Rabelo, D., Coutinho, F.M.B., Barbosa, C.C.R., Rezende, S.M. - Polym. Bull. 34, p. 621 (1995).

8. Coutinho, F.M.B., Neves, M.A.F.S. \& Dias, M.L. - J. Appl. Polym. Sci., 65, p. 1257 (1997).

9. Coutinho, F.M.B., Neves, M.A.F.S., Dias, M.L. \& Alves, G.M. -J. High Resol. Chromatogr., 22 (1), p. 63 (1999).

10. Coutinho, F.M.B Luz, Teixeira, V.G. \& Barbosa, C.C.R. - J. Appl. Polym. Sci., 67, p. 781 (1998).

11. Luz, C.T.L. \& Coutinho, F.M.B. - Eur. Polym. J., 36, p. 547 (2000).

12. ASTM D I895-69 - "Apparent density, bulk factor and pourability of plastic materials”, Annual Book of ASTM, Part. 35, Philadelphia (1975).
13. Figueiredo, J.L. \& Ribeiro, F.R. - "Catálise Heterogênea”, Fundação Caloustre Gulbenkian, Lisboa (1989).

14. Coutinho, F.M.B., Barbosa, C.C.R., Rezende, S.M. Eur. Polym. J., 31 (12), p. 1243 (1995).

15. Rabelo, D. \& Coutinho, F.M.B. - Polym. Bull., 30, p. 725 (1993).

16. Nyhus, A.K., Hagen, S. \& Berge, A. - J. Appl. Polym. Sci., 76, 152 (2000).

17. Kunin, R. - "Ion exchange resins", Robert Krieger Plublishing, New York (1972).

18. Vogel, A.I. - "A text-book of pratical organic chemistry”, in: Qualitative Organic Analysis, Longmans (ed), London (1956).

19. Van Krevelen, D.W., "Properties of polymers", Elsevier, Amesterdan (1994).

20. Rabelo, D. \& Coutinho, F.M.B. - Macromol. Symp., 84, 341 (1994).

21. Silverstein, R.M., Bassler, G.C., Morrill, T.C., "Spectrometric Identification of Organic Compounds", John Wiley, Singapura (1991).

22. Balcerowiak, W. - React Func. Polym., 33, 323 (1997).

23. Hatakeyama, T., Quinn, F.X., "Thermal Analysis: Fundamentals and Applications to Polymer Science", John Wiley \& Sons, New York (1997).

24. Bothe, N, Döscher, F., Klein, J., Widdecke, H. - Polym., 20, 850 (1979).

25. Luz, C.T.L., "Estudo da influência de diluentes dos monômeros na formação da estrutura porosa de resinas de troca iônica à base de 2-vinilpiridina e divinilbenzeno", Tese de Mestrado, Universidade Federal do Rio de Janeiro, 1991.

Recebido: $31 / 08 / 01$ Aprovado: 28/12/01 